\title{
Mielolipoma hepático en una hernia diafragmática peritoneo-pericárdica en un gato: Reporte de caso
}

\author{
Hepatic myelolipoma in a peritoneopericardial diaphragmatic hernia in a cat: case report \\ C Beato ${ }^{\mathrm{a}}, \mathrm{J}$ Smyth ${ }^{\mathrm{b}}$, FR Carvallo ${ }^{\mathrm{c} *}$ \\ aDepartamento de Patología Animal, Facultad de Ciencias Veterinarias y Pecuarias Universidad de Chile, Santiago, Chile. \\ ${ }^{b}$ Department of Pathobiology and Veterinary Science, University of Connecticut, Connecticut, USA. \\ 'Escuela de Medicina Veterinaria, Universidad Santo Tomás, Talca, Chile.
}

\begin{abstract}
SUMMARY
A 10.5-year-old cat was received with sudden anorexia and dyspnea. Ultrasonographic examination of the thoracic cavity revealed that the heart was compressed by a mass on its right aspect. At necropsy, a peritoneo-pericardial diaphragmatic hernia was found, and a portion of the liver and omentum were found within the pericardial sac. Histopathology of the herniated liver revealed the presence of a neoplastic mass characterized by numerous adipocytes, myeloid and erythroid progenitor cells including megakaryocytes and bone spicules (myelolipoma). Peritoneo-pericardial diaphragmatic herniation of the liver is usually an asymptomatic congenital defect in cats. It is considered as an incidental finding and it is frequently associated with hepatic myelolipoma in feline species.
\end{abstract}

Key words: cats, congenital defect, osseus metaplasia, myelolipoma.

\section{RESUMEN}

Se presenta un gato de 10,5 años de edad con anorexia repentina y disnea. Al examen ultrasonográfico de tórax se detectó una masa que comprimía el corazón en el lado derecho. A la necropsia se observó una hernia diafragmática peritoneo pericárdica, misma que contenía segmentos de omento e hígado atrapados dentro del saco pericárdico. Al análisis microscópico, se observó un tejido de neoformación en el segmento de hígado herniado, el cual presentaba un gran número de adipocitos, megacariocitos y células progenitoras mieloides y eritroides, además de pequeños fragmentos óseos. La hernia diafragmática peritoneo pericárdica con atrapamiento hepático es un defecto congénito generalmente asintomático en gatos. Su hallazgo suele ser incidental, y se ha observado que generalmente se asocia al mielolipoma hepático en felinos.

Palabras clave: gatos, malformaciones congénitas, metaplasia ósea, mielolipoma.

\section{INTRODUCCIÓN}

El mielolipoma es una neoplasia benigna compuesta de una mezcla de tejido adiposo bien diferenciado y tejido hematopoyético similar al de la medula ósea (Lack 2000). El origen de este tumor es desconocido, sin embargo se ha sugerido que la proliferación clonal de células madres pluripotentes tiene relación con su presentación (Chang y col2002, Bishop y col 2006). Esta neoplasia se ha descrito en las glándulas adrenales, bazo e hígado de humanos de edad avanzada, primates no humanos, felinos, caninos, ganado y otras especies (Bovo y col 2007, Capen 2007). No obstante, se observa con muy baja frecuencia en animales domésticos, puede surgir como una lesión única o multifocal, y a la fecha no se han reportado casos de metástasis (Kamiie y col 2009).

Aceptado: 28.03.2013.

* Avenida Carlos Schorr 255, Talca, Chile; francisco.carvallo@ gmail.com
A continuación se presenta un caso de un gato con una hernia diafragmática peritoneo-pericárdica, con atrapamiento de un segmento de hígado, el que presentó una lesión nodular en la porción herniada, diagnosticado histopatológicamente como mielolipoma.

\section{MATERIAL Y MÉTODOS}

\section{DESCRIPCIÓN DEL CASO}

Se trata de un gato Ragdoll macho castrado, de 10.5 años de edad. El paciente llegó a la clínica veterinaria presentando disnea y un historial de diabetes mellitus no tratada. A partir del momento de la consulta, el paciente fue tratado con glipizide a dosis de $2.5 \mathrm{mg}$ totales cada 12 horas, prescrito de por vida. El paciente se mantuvo bien por un periodo de 1.5 años, después del cual, repentinamente comenzó a presentar anorexia y disnea. Producto de lo anterior, se realizó un examen ultrasonográfico de la cavidad torácica, en el que se detectó una masa presente 
en el interior del saco pericárdico, que comprimía el corazón. Por decisión del dueño el paciente fue eutanasiado.

\section{NECROPSIA E HISTOPATOLOGÍA}

Se procede a la realización de una necropsia según el procedimiento descrito por Van Kruiningen (1971). Muestras de pulmón, corazón, hígado, bazo, riñón, páncreas, estómago, intestino delgado, intestino grueso, glándula adrenal y cerebro fueron fijados en formalina tamponada al $10 \%$, luego procesados rutinariamente e incluidas en parafina. Secciones de $5 \mu \mathrm{m}$ fueron teñidas con hematoxilina y eosina.

\section{RESULTADOS Y DISCUSIÓN}

En la necropsia, se observó que aproximadamente un 5\% del hígado, principalmente el lóbulo craneal izquierdo, y un segmento del omento con tejido adiposo, se encontraban herniados dentro del saco pericárdico, comprimiendo el corazón (figura 1). La porción del hígado contenida dentro del saco pericárdico medía 5 x 4 $\mathrm{x} 3,5 \mathrm{~cm}$., y presentaba un aspecto moteado amarillo y rojo, con bordes redondeados. El resto del hígado estaba localizado en la cavidad abdominal, y se encontraba aumentado de volumen y friable, con áreas de coloración amarillas y rojas.

Microscópicamente, las secciones del hígado herniado evidenciaban un tejido de neoformación bien delimitado, de bordes irregulares, no encapsulado, compuesto por un gran número de adipocitos mezclados con células progenitoras mieloides y eritroides, incluidos numerosos megacariocitos (figura 2). Además se observaron pequeños fragmentos óseos en la lesión (figura 3). En los már-

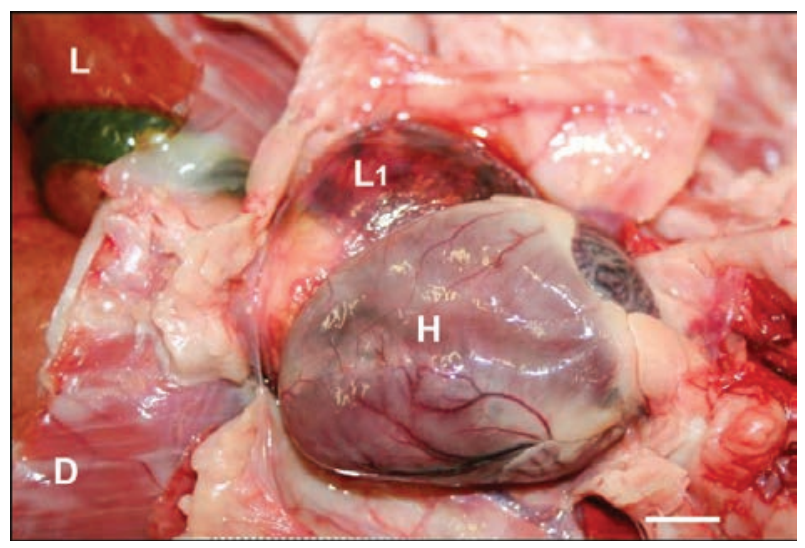

Figura 1. Saco pericárdico conteniendo segmento herniado de hígado comprimiendo el corazón. D: Diafragma; L: Hígado; L1: Segmento herniado de hígado; H: Corazón. Barra: $1 \mathrm{~cm}$.

Pericardial sac containing the herniated segment of the liver compressing the heart. D: Diaphragm, L: Liver; L1: Herniated portion of the liver; H: Heart. Bar: $1 \mathrm{~cm}$.

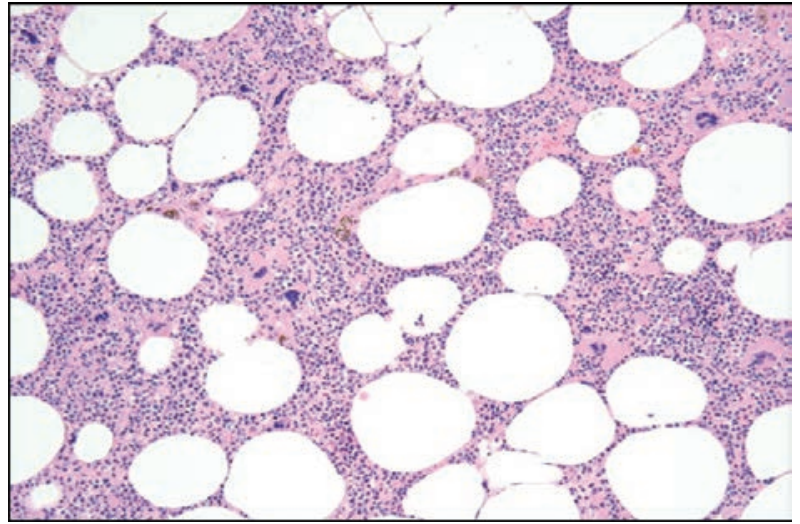

Figura 2. Sección histológica de segmento herniado de hígado en saco pericárdico. Tejido de neoformación compuesto por adipocitos, megacariocitos y células progenitoras mieloides y eritroides. Hematoxilina y Eosina, 200X.

Histologic section of the herniated portion of the liver in the pericardial sac. Tumor mass composed of adipocytes, megakaryocytes, and myeloid and erythroid precursors cells. Hematoxylin and Eosin, 200X.

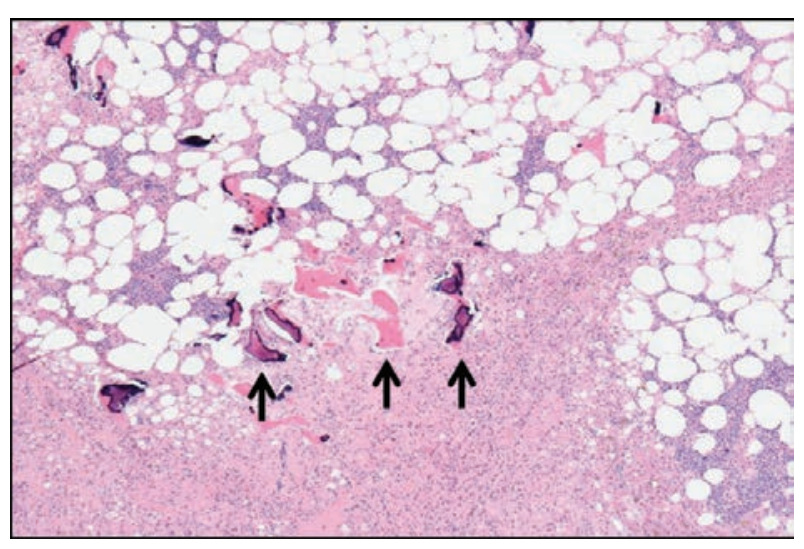

Figura 3. Sección histológica de segmento herniado de hígado en saco pericárdico. Presencia de fragmentos óseos (flechas) en los bordes del tejido de neoformación hepático. Hematoxilina y Eosina, 100X.

Histologic section of the herniated portion of the liver in the pericardial sac. Bone spicules (arrows) at the margins of the hepatic tumor mass. Hematoxylin and Eosin, 100X.

genes de la neoplasia, los cordones hepáticos se observaban atróficos, mientras que los hepatocitos del resto de la muestra presentaban cantidades variables de vacuolas lipídicas dentro del citoplasma. Los sinusoides hepáticos se encontraban focalmente distendidos. En los otros órganos examinados, se evidenció amiloidosis severa de los islotes de Langerhans y una glomerulopatía membranoproliferativa.

Basado en los hallazgos anteriormente descritos, el diagnóstico fue mielolipoma en un lóbulo hepático contenido dentro una hernia diafragmática peritoneo pericárdica. 
En gatos, el mielolipoma es una neoplasia muy poco frecuente, existiendo pocos reportes de casos en la literatura. En esta especie, esta lesión se localiza principalmente en el hígado, y con menor frecuencia en el bazo (Sander y Langham 1972, McCaw y col 1990). La presentación hepática, en conjunto con la presencia de hernia diafragmática peritoneo-pericárdica pareciera ser común (Schuh 1987). Por su parte, en un estudio realizado en 109 felinos silvestres en cautiverio, el guepardo destacó como la especie más afectada con esta neoplasia, con una incidencia del 6\%, (Lombard y col 1968). En humanos y primates no humanos, el mielolipoma se presenta más comúnmente en la glándula suprarrenal (Porter y col 2004, Bovo y col 2007), mientras que en el perro se ubica de preferencia en el bazo (Spangler y col 1994).

Debido a la presencia de tejido hematopoyético en el hígado, de aspecto similar al de la médula ósea, se debe considerar la hematopoyesis extramedular como diagnóstico diferencial. Esta alteración corresponde a la producción de glóbulos rojos fuera de la médula ósea cuando ésta última no es capaz de satisfacer las demandas del organismo. Cabe destacar que la proliferación de elementos hematopoyéticos extra medulares se produce en tres circunstancias: 1) debido a un atrapamiento embriológico de células hematopoyéticas que crecen y proliferan extra medularmente. 2) producto de factores de crecimiento hematopoyético producidos por una neoplasia o tejido dañado, y 3) asociado a trastornos mieloproliferativos o enfermedades hemolíticas que estimulan la formación de focos de hematopoyesis extramedular (Gupta y col 2004, Wouda y col 2010).

Por su parte, las hernias diafragmáticas congénitas se consideran poco frecuentes en animales domésticos (Thrall 1994). No obstante, dentro de este tipo de defectos diafragmáticos, la hernia peritoneo-pericárdica es el tipo más comúnmente observado en perros y gatos (Levine 1987). La embriogénesis de este tipo de hernia es desconocida, sin embargo, se plantea que puede incluir malformaciones o traumas del septo transverso y los pliegues pleuroperitoneales (Noden y Lahunta 1985). En gatos, se ha observado que de todos los órganos abdominales, el hígado es uno de los más propensos a herniarse diafragmáticamente (Garson y col 1980). En los casos de hernia diafragmática peritoneo-pericárdica, es común observar cambios degenerativos en los hepatocitos del segmento de hígado herniado. Se cree que la causa de esta generación celular estaría relacionada con la hipoxia crónica extrema generada por el atrapamiento del hígado en el saco pericárdico, que actuaría como agente inductor en la formación de los mielolipomas en el hígado de estos pacientes (Schuh 1987).

La presencia de formaciones óseas en mielolipomas, ha sido descrita en la presentación adrenal de humanos y otros mamíferos (Lack 2000, Capen 2007). Aunque el origen de estas formaciones es incierto, parecen provenir desde una transformación metaplásica de células de la corteza adrenal o células que revisten los sinusoides adrenales (Capen 2002). En general, entre las hipótesis de la presencia de espículas de hueso en los bordes de una neoplasia se destaca 1) un cambio metaplásico como una reacción de reparación frente a isquemia, necrosis, inflamación o cambios degenerativos en el tumor o tejidos periféricos, y 2) la producción de hueso por células tumorales (Cribbs y col 1999). En el presente caso, se evidenció la presencia de hueso en los bordes de la neoplasia, lo que podría corresponder a la producción de matriz colagenosa densa por células mesenquimáticas y organización en hueso en células del estroma, sin haber relación con una desdiferenciación de células tumorales. Los fibroblastos de células mesenquimáticas forman fibras de colágeno y diferencian en osteoblastos, las que forman las estructuras óseas lamelares.

Generalmente, el diagnóstico de este tipo de hernias es incidental, no obstante, en algunos casos pueden generar signos respiratorios o gastrointestinales (Evans y Biery 1980). En el presente caso, el paciente presentó disnea en la primera consulta, y anorexia repentina en la segunda, sin embargo, estos signos son inespecíficos, y podrían no asociarse con este cuadro clínico en particular.

El tratamiento de estos pacientes es quirúrgico, y la herniorrafia es el procedimiento de elección en todos los casos de hernia diafragmática, debido al potencial desplazamiento de órganos abdominales hacia la cavidad torácica (White y col 2003). La lobectomía hepática también se recomienda en los casos de herniación de algún segmento de hígado (Tahmm, 2001). En general, el pronóstico postquirúrgico de estos pacientes es excelente, presentan un tiempo de sobrevida prolongado y los casos de recurrencia son prácticamente nulos (Wallace y col 1992).

A modo de conclusión, es importante destacar que el mielolipoma hepático es una neoplasia de baja presentación en animales domésticos. Si bien, este caso corresponde a uno de los pocos reportes que existen sobre la presentación de esta neoplasia en conjunto con una hernia diafragmática, no es de extrañar su presentación en gatos, ya que de acuerdo a lo observado en la literatura, esta lesión pareciera presentarse de forma común en felinos domésticos y silvestres.

\section{REFERENCIAS}

Bishop E, JN Eble, L Cheng, M Wang, D Chase, A Orazi, D O'malley. 2006. Adrenal myelolipomas show nonrandom $\mathrm{X}$-chromosome inactivation in hematopoietic elements and fat: support for a clonal origin of myelolipomas. Am J Surg Pathol 30, 838-843.

Bovo G, SC Picozzi, P Vigano, A Giuberti, M Casu, V Manganini, L Mazza, GR Strada. 2007. Giant adrenal myelolipoma: report of a case and review of the literature. Minerva Urol Nefrol 59, 455-458.

Capen C. 2007. Endocrine glands. In: Maxie MG (ed). Pathology of domestic animals. $5^{\text {th }}$ ed. Vol. 3. Saunders, Philadel- 
phia, USA, Pp 325-428.

Chang KC, PI Chen, ZH Huang, YM Lin, P Kuo. 2002. Adrenal myelolipoma with translocation $(3 ; 21)(\mathrm{q} 25 ; \mathrm{p} 11)$. Cancer Genet Cytogen 134, 77-80.

Cribbs RK, M Ishaq, M Arnold, J O’brien, J Lamb, WL Frankel. 1999. Renal cell carcinoma with massive osseus metaplasia and bone marrow elements. Ann Diagn Pathol 3, 294-299.

Evans S, D Biery. 1980. Congenital peritoneopericardial diaphragmatic hernia in the dog and cat: a literature review and 17 additional case histories. Vet Radiology 21, 108116.

Garson H, N Dodman, G Baker. 1980. Diaphragmatic hernia: Analysis of fifty-six cases in dogs and cats. J Small Anim Pract 21, 469-481.

Gupta P, A Naran, Y Auh, J Chung. 2004. Focal intrahepatic extramedullary hematopoiesis presenting as fatty lesions. Am J Roentgenol 184, 1031-1032.

Kamiie J, K Fueki, H Amagai, Y Ichikawa, K Shirota. 2009. Multicentric myelolipoma in a dog. Vet Med Sci 71, 371373.

Lack E. 2000. Tumors of the adrenal gland. In: Fletcher CDM (ed). Diagnostic histopathology of tumors. $2^{\text {nd }}$ ed. Vol. 2. Churchill Livingstone, London, UK, Pp 1057-1082.

Levine S. 1987. Diaphragmatic hernia. Vet Clin N Am-Small 17, 411-430.

Lombard LS, HM Fortna, FM Garner, G Brynjolfsson. 1968. Myelolipomas of the liver in captive wild felidae. Pathol Vet 5, 127-134.

McCaw D, J da Silva, D Shaw. 1990. Hepatic myelolipomas in a cat. J Am Vet Med Assoc 197, 243-244.

Noden D, A de Lahunta. 1985. Respiratory system and partitioning of body cavities. In: Noden DM, de Lahunta A (eds). The Embryology of domestic animals: developmen- tal mechanisms and malformations. Williams \& Wilkins, Baltimore, USA, Pp 279-291.

Porter B, S Goens, K Brasky, G Hubbard. 2004. A case report of hepatocellular carcinoma and focal nodular hyperplasia with a myelolipoma in two chimpanzees and a review of spontaneous hepatobiliary tumors in non-human primates. J Med Primatol 33, 38-47.

Sander C, R Langham. 1972. Myelolipoma of the spleen in a cat. J Am Vet Med Assoc 160, 1101-1103.

Schuh J. 1987. Hepatic nodular myelolipomatosis (myelolipomas) associated with a peritoneo-pericardial diaphragmatic hernia in a cat. J Comp Path 97, 231-235.

Spangler W, M Culbertson, P Kass. 1994. Primary mesenchymal (non angiomatous/non lymphomatous) neoplasms occurring in the canine spleen: anatomic classification, immunohistochemistry, and mitotic activity correlated with patient survival. Vet Pathol 31, 37-47.

Thamm D. 2001. Hepatobiliary tumors. In: Small Animal Clinical Oncology. Withrow SJ, MacEwen EG (eds). WB Saunders, Philadelphia, USA, Pp 327-334.

Thrall D. 1994. The diaphragm. In: Textbook of Veterinary Diagnostic Radiology. $2^{\text {nd }}$ ed. WB Saunders, Philadelphia, USA, Pp 271-272.

Van Kruiningen HJ. 1971. Veterinary autopsy procedure. Vet Clin North Am 1, 163-189.

Wallace J, H Mullen, M Lesser. 1992. A technique for surgical correction of peritoneal pericardial diaphragmatic hernia in dogs and cats. J Am Anim Hosp Assoc 28, 503-510.

White J, P Tisdall, J Norris, R Malik. 2003. Diaphragmatic hernia in a cat mimicking a pulmonary Mass. J Feline Med Surg 5, 197-201

Wouda R, M Chalkley, A Frasera, P Mosesa. 2010. Hepatic myelolipoma incarcerated in a peritoneopericardial diaphragmatic hernia in a cat. Aust Vet $J$ 88, 231-235. 